\title{
Surgical considerations for breast-conserving therapy in patients undergoing neoadjuvant chemotherapy for early-stage breast cancer
}

\author{
G. V. Babiera \\ Department of Surgical Oncology, The University of Texas M.D. Anderson Cancer Center, Houston, TX, USA.
}

\begin{abstract}
According to the National Cancer Comprehensive Network guidelines patients with breast tumours $\leqslant 1 \mathrm{~cm}$ should be offered systemic therapy. Multiple studies, however, have demonstrated no survival advantage between giving chemotherapy before (neoadjuvant) or after (adjuvant) surgery. There are, however, certain benefits that can be derived from patients who undergo neoadjuvant chemotherapy. Such benefits include better stratification of the patient's prognosis based on tumour and nodal response, and converting patients from mastectomy-only candidates to breast-conserving surgery candidates. Due to these advantages, many patients with early-stage breast cancer will undergo neoadjuvant chemotherapy and the surgical issues must be considered for patients who are treated in such a manner to guarantee optimal outcomes. Issues that will be discussed are the surgical preoperative evaluation, extent and timing of local resection of both the breast and regional nodes and finally, the potential future effects of this multi-modality therapy.
\end{abstract}

Keywords: Breast cancer; Neoadjuvant chemotherapy; Breast-conserving surgery

\section{Introduction}

Multiple randomized trials have demonstrated that for patients with breast cancer who require chemotherapy, no difference in survival is conferred if chemotherapy is given before (neoadjuvant) or after (adjuvant) surgery [1-6]. However, neoadjuvant chemotherapy for the treatment of breast cancer does have some advantages, such as the potential for converting disease that would otherwise require mastectomy for local control to that which can be treated by breast conservation, improved stratification of patients by prognosis according to tumour and nodal response, and the ability to assess tumour response to different chemotherapeutic regimens. Due to these advantages, neoadjuvant chemotherapy

Correspondence to: G. V. Babiera, M.D, Department of Surgical Oncology, Unit 444, The University of Texas M.D. Anderson Cancer Center, 1515 Holcombe Boulevard, Houston, TX 77030, USA. Tel: +1713745 1563; Fax: +17137454926

Publication date 25/02/05

BCO/257/2004/FO can be considered for some patients with operable breast cancer who we know would undergo adjuvant chemotherapy based on tumour and nodal status determined pre-operatively. Herein I discuss the surgical considerations involved in managing breast cancer in these patients.

\section{Evaluation of patients being considered for neoadjuvant chemotherapy}

A diagnosis of invasive carcinoma of the breast should be confirmed followed by an accurate assessment of the clinical stage. The basis for establishing candidacy for neoadjuvant chemotherapy should be the result of clinical and pathological examination. Furthermore, six questions should be asked if neoadjuvant chemotherapy is being contemplated.

1. Could giving neoadjuvant chemotherapy convert the required surgical treatment from mastectomy to breast conservation? 
2. Can the patient be followed over time to monitor clinical response to chemotherapy, and, based on the tumour histology, is a response to chemotherapy likely?

3. Can the tumour be identified easily after chemotherapy?

4. How much tissue should be removed if the tumour shows a clinical response?

5. How will the regional nodes be evaluated for metastases?

6. Is there an intellectual benefit derived by the clinician, such as the patient participating in clinical protocols that measure improvement in pathological response, conversion rates from mastectomy to breast-conservation therapy, mechanisms of response, changes in molecular markers and survival outcomes by giving neoadjuvant chemotherapy?

\section{Assessment of clinical stage}

The clinical disease stage should be determined after a thorough medical history, physical and radiographic examinations, preferably by a multidisciplinary group consisting of a radiologist and medical, surgical and radiation oncologists. Tumour size, skin involvement (e.g. ulceration, peau d'orange, erythema, oedema, dimpling), nipple involvement and any suspicious regional adenopathy should be documented.

The use of mammography to determine the extent of disease is vital, as the presence of diffuse or suspicious calcifications may be a contraindication for breast-conservation therapy (Table 1). Additional sonographic imaging of the breast to identify other foci of cancer and documentation of the extent of regional adenopathy by fine-needle aspiration is also very helpful [7]. The benefit of magnetic resonance imaging (MRI) as a screening tool for breast cancer has not been proven in a large, randomized setting $[8,9]$. However, limited data are emerging that MRI is an effective adjunct to mammography and sonography for the evaluation of patients at high risk for breast cancer $[10,11]$. Currently, positron emission tomography (PET) is not used for detecting primary breast cancers because of its inability to visualize small malignancies [12].

\section{Cosmetic benefits of neoadjuvant chemotherapy}

Breast-conservation therapy with optimal cosmetic results may not be possible for patients with relatively large tumours and small breasts; mastectomy might be a better choice for such patients. However, the ability of neoadjuvant chemotherapy to shrink tumours may allow patients who would otherwise
Table 1. Indications for breast-conservation therapy for early-stage breast cancer after neoadjuvant chemotherapy.

Desire for breast-conservation

Ability to undergo radiation therapy

Absence of diffuse suspicious microcalcifications

Absence of multicentric disease

require mastectomy to undergo breast conservation instead. Furthermore, patients who are candidates for breast conservation to begin with may derive additional cosmetic benefit from undergoing neoadjuvant chemotherapy because of the potential for removing still smaller amounts of tissue as the amount of tissue removed is inversely proportional to the cosmetic outcome [13].

The feasibility of breast-conservation surgery after neoadjuvant chemotherapy for large, operable breast cancers has been confirmed in many studies (Table 2). However, attempts to achieve better cosmetic outcome by giving neoadjuvant chemotherapy followed by excision of the smallest possible amount of tissue from the breast raises concern regarding increased rates of local failure. Several studies have shown that local failure rates were not high when local treatment included surgery and radiation therapy after neoadjuvant chemotherapy $[1,2,4,14,15]$. The relatively high local recurrence rates of $13 \%$ [16], 27\% [17] and $28 \%$ [18] reported in these studies reflected the use of radiation as the only local treatment after neoadjuvant chemotherapy.

\section{Assessment of clinical response}

To determine the best time for surgery in conjunction with neoadjuvant therapy, it is important to be able to assess the tumour's clinical response, both by physical examination and by radiography. Appropriate tumour markers such as metallic clips should be placed before initiating neoadjuvant chemotherapy if the tumour is $\leqslant 2 \mathrm{~cm}$ in diameter [19]. If no markers are placed at that time, careful monitoring of the tumour should be performed and a clip marker placed if the tumour appears to be responding well.

Throughout the course of chemotherapy, tumour response is monitored by physical examination after each cycle. Although radiographic imaging is less than optimal for predicting which tumours respond pathologically after neoadjuvant chemotherapy, mammography, sonography, or both should be performed after the fourth cycle of chemotherapy, as the maximum response typically will have occurred at that time for most tumours [20]. The use of MRI [21-23] or PET imaging [24] to monitor response seems promising, although their sensitivity remains to be established 
Table 2. Reported rates of breast-conservation after neoadjuvant chemotherapy for patients with large primary and operable breast cancer.

\begin{tabular}{|c|c|c|c|c|c|c|}
\hline Authors & Year & Stage & $n$ & $\begin{array}{l}\text { Breast- } \\
\text { conservation } \\
\text { therapy rate }(\%)\end{array}$ & $\begin{array}{l}\text { Median } \\
\text { follow-up } \\
\text { (months) }\end{array}$ & $\begin{array}{l}\text { Local } \\
\text { recurrence } \\
(\%)\end{array}$ \\
\hline Bonadonna et al. [39] & 1990 & II, III & 161 & 81 & NA & 1 \\
\hline Jacquillat et al. [16] ${ }^{\star}$ & 1990 & I, II, III & 250 & 94 & 62 & 13 \\
\hline Scholl et al. [17] ${ }^{*}$ & 1994 & II, III & 200 & 82 & 54 & 27 \\
\hline Veronesi et al. [14] & 1995 & II, III & 226 & 90 & $36^{\dagger}$ & 6 \\
\hline Powles et al. [1] & 1995 & II & 105 & 88 & 28 & 1 \\
\hline Fisher et al. [4] & 1998 & I, II & 743 & 68 & $72^{\dagger}$ & 8 \\
\hline Bonadonna et al. [40] & 1998 & II, III & 536 & 85 & 65 & 7 \\
\hline Makris et al. [2] & 1998 & II & 149 & 99 & 48 & 3 \\
\hline Mauriac et al. [18] ${ }^{*}$ & 1999 & II, III & 134 & 63 & 124 & 28 \\
\hline
\end{tabular}

in large multicenter trials. If the patient's tumour does not seem to be responding or progressing, surgery followed by pathological analysis may be appropriate to determine the appropriateness of using another non-resistant agent as adjuvant therapy.

Infiltrating lobular carcinoma is a histological variant that appears to respond poorly to neoadjuvant chemotherapy $[25,26]$. In addition, assessing the extent of disease and response to chemotherapy in patients with this type of cancer is notoriously difficult by physical examination or current imaging methods [8]. Both Cocquyt et al. [25] and Mathieu et al. [26] found that the rates of conversion from mastectomy to breast-conservation therapy were much lower for patients with infiltrating lobular carcinoma than for patients with invasive ductal carcinoma. Therefore, neoadjuvant chemotherapy for patients with this type of tumour who are trying to attempt breastconservation therapy by shrinking the tumour may not be worthwhile. However, if acceptable cosmesis can be obtained with or without neoadjuvant chemotherapy, breast-conservation therapy should not be withheld from patients with infiltrating lobular carcinoma, as breast conservation does not seem to produce inferior locoregional recurrence or survival rates relative to mastectomy [25-28]. Despite the lack of pathological response to chemotherapy it is still important that patient's diagnosed with infiltrating lobular carcinoma receive adjuvant or neoadjuvant chemotherapy as this histology may have a different biology than its' more common counterpart, invasive ductal carcinoma.

\section{Localization of tumours}

Depending on the chemotherapeutic regimen used, approximately $7-52 \%$ of patients with breast cancer have a complete clinical response to chemotherapy, whereas only $2-23 \%$ will have a complete pathological response, explaining the need for surgical treatment after neoadjuvant chemotherapy [29]. Placing clips in the tumour bed before the initiation of chemotherapy or early during chemotherapy is important for intraoperative identification of a lesion with wireguided localization both for the surgeon and pathologist. Failure to place a localization marker before chemotherapy is begun may result in a need for mastectomy after neoadjuvant chemotherapy if the lesion cannot be identified with any imaging method or the location of the lesion cannot be reconstructed accurately from previously acquired radiographic images.

\section{Volume of tissue resected}

The question of how much tissue should be resected intra-operatively in the event of a partial or complete response after chemotherapy (i.e. the size of the original tumour or only gross residual disease) remains controversial, largely because tumours that respond to chemotherapy may not shrink uniformly. In the randomized National Surgical Adjuvant Breast and Bowel Project B-18 investigating preoperative chemotherapy for patients with operable breast cancer, the rate of ipsilateral breast tumour recurrence was $15.9 \%$ among patients converted from mastectomy to breast-conservation therapy; however, the rate of recurrence was only $9.9 \%$ among patients who were initial candidates for breast-conservation therapy. Whether this difference in recurrence rates resulted from residual disease being left behind or from differences in age and initial tumour size, as explained by Wolmark et al. [5], remains unclear. No definitive studies have been performed to determine how much tissue should be resected in the event 
of an apparent clinical response to chemotherapy. However, the general recommendation is that all gross evidence of disease be removed, including any associated suspicious or documented cancerous calcifications. Particular attention should be paid to the final pathological and margin assessment to determine whether additional surgery is needed.

\section{Axillary evaluation}

If very little definitive information is known regarding the extent of resection of the primary tumour after neoadjuvant chemotherapy, much less is known about what to do with the regional nodes in patients who have undergone neoadjuvant chemotherapy. For example, should sentinel lymph node biopsy or axillary lymph node dissection be done before or after the initiation of chemotherapy? Although sentinel lymph node biopsy is not yet the standard of care throughout the nation, it is routinely used for regional node evaluation in patients with clinically nodenegative early-stage breast cancer who undergo surgery first. However, the role of sentinel lymph node biopsy after neoadjuvant chemotherapy is still controversial. Several studies, mainly from single institutions, have found varying identification (84.3-93.5\%) and false-negative (0-33\%) rates in patients who underwent sentinel lymph node biopsy after neoadjuvant chemotherapy [30-36]. In the only multicenter trial to indirectly investigate the identification and falsenegative rates in patients who underwent sentinel lymph node biopsy after neoadjuvant chemotherapy, Mamounas [37] reported an identification rate of $77 \%$ and false-negative rate of $14 \%$.

Should sentinel lymph node biopsy be performed before or after neoadjuvant chemotherapy? The variability in identification and false-negative rates in patients who have undergone sentinel lymph node biopsy after neoadjuvant chemotherapy led Sabel et al. [38] to propose that sentinel lymph node biopsy might be more valuable if performed before rather than after such therapy. The advantages of this approach are more accurate disease staging, more selective use of taxanes for patients with nodepositive disease, and better focused adjuvant radiation therapy based on nodal information obtained from the biopsy procedure. However, the disadvantages are the delay in chemotherapy, the need for more axillary lymph node dissections, the assumption that the sentinel lymph node biopsy analysis before initiation of neoadjuvant chemotherapy, if negative for metastasis is accurate after neoadjuvant chemotherapy, and most importantly, the need for at least two operations.

No consensus has been reached regarding the timing of sentinel lymph node biopsy as related to neoadjuvant chemotherapy because of a lack of data from randomized prospective studies. However, axillary lymph node dissection performed after neoadjuvant chemotherapy for patients found to have pathologically node-positive breast cancer before the initiation of neoadjuvant chemotherapy probably remains the standard. Conceptual studies investigating the possibility of performing sentinel lymph node biopsy after neoadjuvant chemotherapy for patients found to have node-positive disease before neoadjuvant chemotherapy are being explored by the American College of Surgeons Oncology Group.

\section{Research endeavors}

The most intriguing aspect of neoadjuvant chemotherapy is the possible benefit derived from assessment of tumour response to the chemotherapy, as the pathological response appears to be associated with the patient's prognosis. For example, designing trials for patients whose tumours seem to respond poorly to standard chemotherapeutic regimens by assessing tumour response would seem to benefit from such information. Agents such as growth factor inhibitors, antihormonal therapies, angiogenesis inhibitors and antisense oligonucleotides, tested in clinical trials, could be beneficial for patients whose tumours show a poor response to cytotoxic chemotherapy and potentially improve survival. Furthermore, by assessing tumour response based on chemotherapeutic regimen, we may be able to predict the success of breast-conservation therapy or avoid surgery altogether if a certain regimen is demonstrating in a majority of patients a complete pathological response after neoadjuvant chemotherapy.

\section{Conclusion}

The primary role of the surgeon in the treatment of a patient with breast cancer who undergoes neoadjuvant chemotherapy is to establish a balance between a good cosmetic outcome and limiting the risk of locoregional recurrence. The surgeon should discuss with the patient issues such as the need for compliance with radiation therapy and long-term follow-up, the possibility of re-excision or failure to convert to breast-conservation therapy, and to address the possibilities of a complete pathological response after surgery. Also, it is imperative that a localizing marker be placed in the tumour bed to facilitate identification of the original tumour site in the event of a clinical response to neoadjuvant chemotherapy. It appears that sentinel lymph node biopsy for clinically node negative patients can be performed after the initiation of chemotherapy. However, surgeons have not 
reached a consensus regarding the role of sentinel lymph node biopsy after neoadjuvant chemotherapy for known node-positive disease before initiating chemotherapy nor the timing of sentinel lymph node biopsy because of a lack of data on these issues. The standard at this point in time is to perform an axillary lymph node dissection for patients who are known to be clinically node positive. Finally, the main advantage of neoadjuvant chemotherapy is the ability to assess tumour response to different chemotherapeutic regimens. It is hoped that the use of this information will lead to the development and use of novel agents to improve breastconservation therapy conversion rates, potentially, avoid surgery altogether and ultimately, to improve survival for patients with breast cancer.

\section{References}

1. Powles TJ, Hickish TF, Makris A, et al. Randomized trial of chemoendocrine therapy started before or after surgery for treatment of primary breast cancer. J Clin Oncol 1995; 13: 547-552.

2. Makris A, Powles TJ, Ashley SE, et al. A reduction in the requirements for mastectomy in a randomized trial of neoadjuvant chemoendocrine therapy in primary breast cancer. Ann Oncol 1998; 9: 1179-1184.

3. Fisher B, Brown A, Mamounas E, et al. Effect of preoperative chemotherapy on local-regional disease in women with operable breast cancer: findings from National Surgical Adjuvant Breast and Bowel Project B-18. J Clin Oncol 1997; 15: 2483-2493.

4. Fisher B, Bryant J, Wolmark N, et al. Effect of preoperative chemotherapy on the outcome of women with operable breast cancer. J Clin Oncol 1998; 16: 2672-2685.

5. Wolmark N, Wang J, Mamounas E, et al. Preoperative chemotherapy in patients with operable breast cancer: nine-year results from National Surgical Adjuvant Breast and Bowel Project B-18. J Natl Cancer Inst Monogr 2001; 30: 96-102.

6. van der Hage JA, van de Velde CJ, Julien JP, et al. Preoperative chemotherapy in primary operable breast cancer: results from the European Organization for Research and Treatment of Cancer trial 10902. J Clin Oncol 2001; 19: 4224-4237.

7. Bedrosian I, Bedi D, Kuerer HM, et al. Impact of clinicopathological factors on sensitivity of axillary ultrasonography in the detection of axillary nodal metastases in patients with breast cancer. Ann Surg Oncol 2003; 10: 1025-1030.

8. Orel SG, Schnall MD. MR imaging of the breast for the detection, diagnosis, and staging of breast cancer. Radiology 2001; 220: 13-30.

9. Esserman L, Hylton N, Yassa L, et al. Utility of magnetic resonance imaging in the management of breast cancer: evidence for improved preoperative staging. J Clin Oncol 1999; 17: 110-119.

10. Hata T, Takahashi H, Watanabe K, et al. Magnetic resonance imaging for preoperative evaluation of breast cancer: a comparative study with mammography and ultrasonography. J Am Coll Surg 2004; 198: 190-197.
11. Kriege M, Brekelmans CT, Boetes C, et al. Efficacy of $\mathrm{MRI}$ and mammography for breast-cancer screening in women with a familial or genetic predisposition. New Engl J Med 2004; 351: 427-437.

12. Rose C, Dose J, Avril N. Positron emission tomography for the diagnosis of breast cancer. Nucl Med Commun 2002; 23: 613-618.

13. Taylor ME, Perez CA, Halverson KJ, et al. Factors influencing cosmetic results after conservation therapy for breast cancer. Int J Radiat Oncol Biol Phys 1995; 31: 753-764.

14. Veronesi U, Bonadonna G, Zurrida S, et al. Conservation surgery after primary chemotherapy in large carcinomas of the breast. Ann Surg 1995; 222: 612-618.

15. Bonadonna G, Valagussa $P$, Zucali $R$, et al. Primary chemotherapy in surgically resectable breast cancer. CA-Cancer J Clin 1995; 45: 227-243.

16. Jacquillat $C$, Weil $M$, Baillet $F$, et al. Results of neoadjuvant chemotherapy and radiation therapy in the breast-conserving treatment of 250 patients with all stages of infiltrative breast cancer. Cancer 1990; 66: 119-129.

17. Scholl SM, Fourquet A, Asselain B, et al. Neoadjuvant versus adjuvant chemotherapy in premenopausal patients with tumours considered too large for breast conserving surgery: preliminary results of a randomised trial: S6. Eur J Cancer 1994; 30A: 645-652.

18. Mauriac L, MacGrogan G, Avril A, et al. Neoadjuvant chemotherapy for operable breast carcinoma larger than $3 \mathrm{~cm}$ : a unicentre randomized trial with a 124-month median follow-up. Institut Bergonie Bordeaux Groupe Sein (IBBGS). Ann Oncol 1999; 10: 47-52.

19. Kuerer HM, Beahm EK, Swisher SG, et al. Surgery for inoperable breast cancer. Am J Surg 2002; 183: 160-161.

20. Kuerer HM, Hunt KK, Newman LA, et al. Neoadjuvant chemotherapy in women with invasive breast carcinoma: conceptual basis and fundamental surgical issues. $\mathrm{J} \mathrm{Am}$ Coll Surg 2000; 190: 350-363.

21. Rosen EL, Blackwell KL, Baker JA, et al. Accuracy of MRI in the detection of residual breast cancer after neoadjuvant chemotherapy. Am J Roentgenol 2003; 181: 1275-1282.

22. Delille JP, Slanetz PJ, Yeh ED, et al. Invasive ductal breast carcinoma response to neoadjuvant chemotherapy: noninvasive monitoring with functional MR imaging pilot study. Radiology 2003; 228: 63-69.

23. Partridge SC, Gibbs JE, Lu Y, et al. Accuracy of MR imaging for revealing residual breast cancer in patients who have undergone neoadjuvant chemotherapy. Am J Roentgenol 2002; 179: 1193-1199.

24. Schelling M, Avril N, Nahrig J, et al. Positron emission tomography using [(18)F]fluorodeoxyglucose for monitoring primary chemotherapy in breast cancer. $J$ Clin Oncol 2000; 18: 1689-1695.

25. Cocquyt VF, Blondeel PN, Depypere HT, et al. Different responses to preoperative chemotherapy for invasive lobular and invasive ductal breast carcinoma. Eur J Surg Oncol 2003; 29: 361-367.

26. Mathieu MC, Rouzier R, Llombart-Cussac A, et al. The poor responsiveness of infiltrating lobular breast carcinomas to neoadjuvant chemotherapy can be explained by their biological profile. Eur $J$ Cancer 2004; 40: 342-351.

27. Chung MA, Cole B, Wanebo HJ, et al. Optimal surgical treatment of invasive lobular carcinoma of the breast. Ann Surg Oncol 1997; 4: 545-550. 
28. Bouvet M, Ollila DW, Hunt KK, et al. Role of conservation therapy for invasive lobular carcinoma of the breast. Ann Surg Oncol 1997; 4: 650-654.

29. Mamounas E. Neoadjuvant chemotherapy for operable breast cancer. Diseases of the Breast 2003; 6: 1-20.

30. Breslin TM, Cohen L, Sahin A, et al. Sentinel lymph node biopsy is accurate after neoadjuvant chemotherapy for breast cancer. J Clin Oncol 2000; 18: 3480-3486.

31. Nason KS, Anderson BO, Byrd DR, et al. Increased false negative sentinel node biopsy rates after preoperative chemotherapy for invasive breast carcinoma. Cancer 2000; 89: 2187-2194.

32. Julian TB, Patel N, Dusi D, et al. Sentinel lymph node biopsy after neoadjuvant chemotherapy for breast cancer. Am J Surg 2001; 182: 407-410.

33. Fernandez A, Cortes M, Benito E, et al. Gamma probe sentinel node localization and biopsy in breast cancer patients treated with a neoadjuvant chemotherapy scheme. Nucl Med Commun 2001; 22: 361-366.

34. Stearns V, Ewing CA, Slack R, et al. Sentinel lymphadenectomy after neoadjuvant chemotherapy for breast cancer may reliably represent the axilla except for inflammatory breast cancer. Ann Surg Oncol 2002; 9: 235-242.
35. Haid A, Tausch C, Lang A, et al. Is sentinel lymph node biopsy reliable and indicated after preoperative chemotherapy in patients with breast carcinoma? Cancer 2001; 92: 1080-1084.

36. Miller AR, Thomason VE, Yeh IT, et al. Analysis of sentinel lymph node mapping with immediate pathologic review in patients receiving preoperative chemotherapy for breast carcinoma. Ann Surg Oncol 2002; 9: 243-247.

37. Mamounas E, Brown A, Smith R, et al. Accuracy of sentinel node biopsy after neoadjuvant chemotherapy in breast cancer: updated results from NSABP B-27. [Abstract 140]. Proc Am Soc Clin Oncol 2002; 21: 36 a.

38. Sabel MS, Schott AF, Kleer CG, et al. Sentinel node biopsy prior to neoadjuvant chemotherapy. Am J Surg 2003; 186: 102-105.

39. Bonadonna G, Veronesi U, Brambilla C, et al. Primary chemotherapy to avoid mastectomy in tumours with diameters of three centimeters or more. $J$ Natl Cancer Inst 1990; 82: 1539-1545.

40. Bonadonna G, Valagussa P, Brambilla C, et al. Primary chemotherapy in operable breast cancer: eight-year experience at the Milan Cancer Institute. $J$ Clin Oncol 1998; 16: 93-100. 